\title{
Neutrophil/lymphocyte ratio and Red blood cell distribution width are independent risk factors for 30-day mortality in Gastrointestinal system bleeding patients
}

\author{
ERTUGRUL ALTINBILEK ${ }^{1}$, DERYA OZTURK ${ }^{1}$, CEMIL KAVALCI ${ }^{2}$ \\ 1 Sisli Etfal Training and Research Hospital, Emergency department, İstanbul/Turkey \\ 2 Baskent University Faculty of Medicine, Ankara \\ Corresponding author \\ Prof.Dr.CemilKavalcr \\ BaşkentÜniversitesiTipFakültesiAcilTip AD, Ankara/Turkey \\ cemkavalci@yahoo.com \\ +90312203
}

\section{ABSTRACT}

Background: In this study, we aimed to examine demographic and endoscopic features of patients with GI bleeding to determine the factors affecting 30-day mortality. Method: Patient's demographic features, laboratory outcomes, comorbidities, drug use, endoscopy outcomes, Glasgow-Blatchford scores, and mortality status were examined. The factors affecting 30-day mortality were investigated. Results: The mean age of the patients was $58.2 \pm 17.4$ years, and $72.1 \%$ were male patients. 30-day mortality rate was found to be $14.4 \%$. The mean age of patients who died was high $(\mathrm{p}<0.05)$. The incidence of mortality was high in the presence of comorbidity, malignancy, and cirrhosis $(\mathrm{p}<0.05)$. Systolic blood pressure was low in the patients who died $(\mathrm{p}<0.05)$. No significant correlation was found between mortality and gender, symptoms, predisposing factors, lesion type and Forrest score, diastolic blood pressure and heart rate $(\mathrm{p}>0.05)$. Urea, neutrophils, red blood cell distribution width / platelet ratio, neutrophil / lymphocyte ratio and RDW levels were high, and hemoglobin level was significantly low in patients with a mortal progression $(\mathrm{p}<0.05)$. No significant correlation was found between mortality, and platelet and lymphocyte levels $(p>0.05)$. Glasgow-Blatchford score was significantly higher in patients who died $(\mathrm{p}<0.05)$. Conclusion: Many factors affect 30-day mortality in GI bleeding. It should be remembered that follow-up of patients with an advanced age who have comorbidity and impaired hemodynamics should be kept for long, and that these patients are at a high risk for mortality.
According to our results, NLR and RDW are independent factors that determine the 30-day mortality in upper GI bleeding.

Keywords: emergency, mortality, NLR, RDW

\section{INTRODUCTION}

Patients presenting to emergency departments with upper Gastrointestinal (GI) bleeding constitute a significant percentage of all emergency department admissions. Upper GI bleeding accounts for $80-85 \%$ of all cases of GI bleeding with an incidence of $50-150$ per 100,000 persons (1-3). Notably, $80 \%$ of patients with GI bleeding recover spontaneously without any intervention, and $20 \%$ of patients receive medical or surgical treatment $(2,3)$. Despite advances in endoscopic and medical therapy, the mortality rate associated with this condition is $2-5 \%$ (4-6).

Patients with massive upper GI bleeding may present to emergency departments with shock, as well as with symptomatic anemiasecondary to chronic blood loss. Given the high mortality rate associated with GI bleeding, rapid diagnosis and prompt treatment are important in such cases (7)

Although several factors are known to affect mortality rates in patients with upper GI bleeding, poor endoscopic outcomes and the severity of bleeding are the most important risk factors associated with mortality. The most important prognostic factor remains controversial (1).

We investigated the factors independently affecting 30-day mortality rates in patients with GI bleeding.

\section{MATERIALS AND METHODS}

Following approval from the Local Ethics Committee of ŞişliEtfal Training and Research Hospital, we retrospectively investigated patients who presented to the emergency department.

Patient details were obtained from the electronic medical record system of the hospital. We contacted patients' relatives in cases where the out-of-hospital mortality status of patients was unknown to confirm if those patients had survived and to obtain mortality details in the deceased.

Patient demographics, laboratory test results, comorbidities, drug use, endoscopy outcomes, Glasgow-Blatchford scores and mortality status were examined and factors affecting 30-day mortality rates were investigated.

After screening using the information processing system of the hospital, we included the following patients in the study: patients aged $>18$ years who presented with melena, hematemesis or hematochezia during physical examination in whom bleeding was confirmed to be GI bleeding and patients with GI bleeding definitively diagnosed by endoscopic or colonoscopic examination. The following patients were excluded from the study: patients aged $<18$ years, those with suspected GI bleeding and patients in whom data pertaining to history, physical examination and laboratory tests were missing.

The patients were divided into two groups as those who deceased within 30 days after gastrointestinal bleeding (Group2) and those who survived (Group 1).

Statistical Analysis 
Data were analyzed using the SPSS 22.0 software.

Parametric data were expressed as mean \pm standard deviation. Non-parametric data were expressed as medians and interquartile ranges, and qualitative data were expressed as frequencies and percentages. Normality of variables was evaluated using the Kolmogorov-Smirnov test. The Student t-test was used to analyze parametric data, and the Mann-Whitney U test was used to analyse non-parametric data. The Chi-square and the Fischer exact test were used to analyze qualitative data. Multivariate logistic regression analysis was used to determine independent factors affecting mortality rates. Receiver operating characteristic curve (ROC) analysis was performed to determine the effectiveness of detecting mortality rates for neutrophil/ lymphocyte ratio (NLR) and the red blood cell distribution width (RDW). A p value $<0.05$ was considered statistically significant.

\section{RESULTS}

This study included 111 patients. Notably, 16 patients (14.4\%) died within 30 days of developing upper GI bleeding. Mean age of the patients was $58.2 \pm 17.4$ years and $72.1 \%$ were men (Table 1 ).

The mean age of deceased patients was significantly high $(\mathrm{p}<0.05)$. No significant correlation was observed between mortality rates and sex (Table 1). The most common

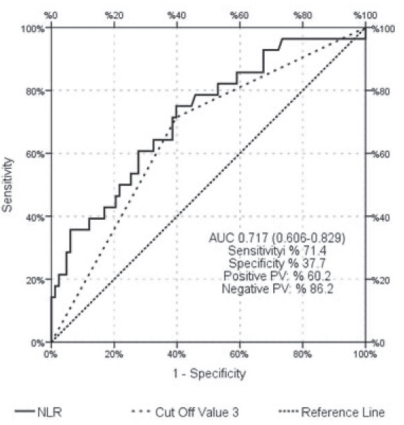

Figure 1. ROC analysis for NLR

List abbreviations and their meaning under the table

NLR:neutrophil/lymphocyte ratio

finding was melena (73\%); however, no significant correlation was observed between mortality rates and all findings (Table2) ( $\mathrm{p}>0.05)$.

Among all patients included in the study, $60.4 \%$ reported comorbidities. The mortality rate was high in patients with comorbidities, malignancy, and cirrhosis $(\mathrm{p}<0.05)$ (Table 1). Predisposing factors were observed in $79.3 \%$ of patients; however, no significant correlation was observed between predisposing factors and mortality rates $(\mathrm{p}>0.05)$ (Table 1$)$. Systolic blood pressure was significantly lower in deceased patients $(\mathrm{p}<0.05)$. No significant correlation was observed between mortality rates and diastolic blood pressure and heart rate $(\mathrm{p}>0.05)$. Serum blood urea nitrogen (BUN) and neutrophil levels, RDW/

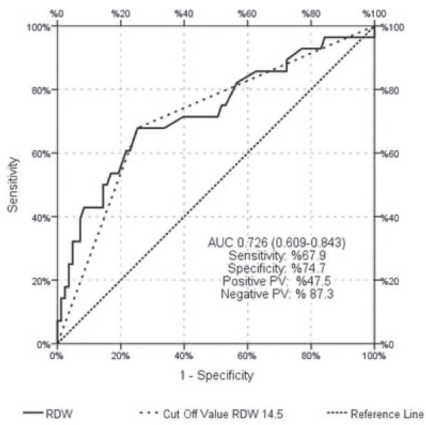

Figure 2. ROC analysis for RDW

List abbreviations and their meaning $R D W$ : red blood cell distribution width

to platelet (PLT) ratio, NLR, and RDW levels were high, and hemoglobin(HGB) level was significantly low in deceased patients $(\mathrm{p}<0.05)$. No significant correlation was observed between mortality rates and serum PLT and lymphocyte levels ( $\mathrm{p}>0.05)$ (Table 3).

The median Glasgow-Blatchford score was 14 (6) in deceased patients and 11 (7) in surviving patients. The median GlasgowBlatchford score was significantly higher in deceased patients $(\mathrm{p}<0.05)$ The most common lesions observed in patients were duodenal and/or peptic ulcers (95.5\%); however, no significant correlation was observed between the type of lesion and mortality rates $(\mathrm{p}>0.05)$. No significant correlation was observed between mortality rates and the Forrest classification $(\mathrm{p}>0.05)$ (Table 4).

Table 1.Demographic and historycal characteristics of patients

\begin{tabular}{|c|c|c|c|c|}
\hline \multicolumn{2}{|l|}{ Variable } & $\begin{array}{l}\text { Survival (n:95) } \\
\text { n (\%) }\end{array}$ & $\begin{array}{l}\text { Deceased (n: 16) } \\
\text { n (\%) }\end{array}$ & P value \\
\hline \multicolumn{2}{|c|}{ Age, Mean \pm SD (years) } & $56.6 \pm 17.8$ & $68.3 \pm 9.9$ & $0.012^{*}$ \\
\hline \multirow[t]{2}{*}{ Gender } & Male & $70(73.7 \%)$ & $10(62.5 \%)$ & 0.999 \\
\hline & Female & $25(26.3 \%)$ & $6(37.5 \%)$ & \\
\hline \multicolumn{2}{|c|}{ Comorbidity } & $52(54.7 \%)$ & $15(93.8 \%)$ & $0.003^{\star}$ \\
\hline \multirow[t]{12}{*}{ History } & Hypertension & $17(17.9 \%)$ & $4(25 \%)$ & 0.499 \\
\hline & Diabetes mellitüs & $13(13.7 \%)$ & $3(18.8 \%)$ & 0.699 \\
\hline & Dementia/Alzheimer & $4(4.2 \%)$ & $1(\%)$ & 0.247 \\
\hline & Stroke & $2(2.1 \%)$ & $1(6.3 \%)$ & 0.376 \\
\hline & Malignancy & $3(3.2 \%)$ & $4(25 \%)$ & $0.008^{\star}$ \\
\hline & Ischemic heart disease, cardiac failure & $28(29.5 \%)$ & $8(50 \%)$ & 0.105 \\
\hline & Cirrhosis & $11(11.6 \%)$ & $5(31.3 \%)$ & $0.038^{\star}$ \\
\hline & Varices/Gastritis & $24(25.3 \%)$ & $7(43.8 \%)$ & 0.141 \\
\hline & Non steroidal antiinflammatory & $26(27.4 \%)$ & $1(6.3 \%)$ & 0.112 \\
\hline & Antiaggregant/anticoagulant & $21(22.1 \%)$ & $7(43.8 \%)$ & 0.115 \\
\hline & Alcohol & $5(5.3 \%)$ & $0(0 \%)$ & 0.999 \\
\hline & Previous bleeding & $39(41.1 \%)$ & $6(37.5 \%)$ & 0.999 \\
\hline
\end{tabular}


Table 2.Complaint and finding of patients at admission according to groups

\begin{tabular}{|c|c|c|c|c|}
\hline Variable & & Survival n (\%) & Deceased n (\%) & P value \\
\hline \multirow[t]{7}{*}{ Complaint } & Melena & $70(73.7 \%)$ & $11(68.8 \%)$ & 0.762 \\
\hline & Hematemesis & $32(33.7 \%)$ & $6(37.5 \%)$ & 0.781 \\
\hline & Hematochezia & $18(18.9 \%)$ & $4(25 \%)$ & 0.518 \\
\hline & Dizziness & $6(6.30 \%)$ & $0(0 \%)$ & 0.591 \\
\hline & Sweating & $2(2.1 \%)$ & $0(0 \%)$ & 0.999 \\
\hline & Syncope & $11(11.6 \%)$ & $5(31.3 \%)$ & 0.054 \\
\hline & Weakness & $18(18.9 \%)$ & $4(25 \%)$ & 0.518 \\
\hline \multirow[t]{7}{*}{ Finding } & Melena & $70(73.7 \%)$ & $11(68.8 \%)$ & 0.762 \\
\hline & Hematemesis & $32(33.7 \%)$ & $6(37.5 \%)$ & 0.781 \\
\hline & Hematochezia & $18(18.9 \%)$ & $4(25 \%)$ & 0.518 \\
\hline & Dizziness & $6(6.30 \%)$ & $0(0 \%)$ & 0.591 \\
\hline & Sweating & $2(2.1 \%)$ & $0(0 \%)$ & 0.999 \\
\hline & Syncope & $11(11.6 \%)$ & $5(31.3 \%)$ & 0.054 \\
\hline & Weakness & $18(18.9 \%)$ & $4(25 \%)$ & 0.518 \\
\hline
\end{tabular}

Table 3. Vital sign and labratory results of patient according to groups

\begin{tabular}{lllll}
\hline Variable & & Survival (n:95) & Deceased (n: 16) & P value \\
\hline Vital sign & Systolic blood pressure, Median (IQR) (mmHg) & $110(20)$ & $97.5(25)$ & 0.004 \\
& Diastolic blood pressure, Median (IQR) (mmHg) & $70(14)$ & $60(23)$ & 0.075 \\
& Pulse, Median (IQR) (beats/min) & $80(28)$ & $90(33)$ & 0.089 \\
\hline Labratory results & UREA, Median (IQR) (mg/dL) & $60(44)$ & $76.9(61)$ & $0.024^{*}$ \\
& HGB, Mean \pm SD (gr/dl) & $10 \pm 2.7$ & $8.3 \pm 2.9$ & $0.042^{*}$ \\
& Platelet, Median (IQR) (x103/mm3) & $212(90)$ & $175.5(206.3)$ & 0.247 \\
& RDW, Median (IQR) & $13.7(2)$ & $16.2(4)$ & $<0.001$ \\
& Neutrophils, Median (IQR) (x103/mm3) & $6.2(4)$ & $8(7)$ & 0.017 \\
& Lymphocytes, Mean \pm SD (x103/mm3) & $2.2 \pm 1.1$ & $1.9 \pm 1.5$ & 0.281 \\
\hline
\end{tabular}

IQR: Interquartile range, HGB: Heamoglobin, RDW: red blood cell distribution width

Table 4. Endoscopy results and Forest clasification according to groups.

\begin{tabular}{lllll}
\hline Categorical & & Survival, n(\%) & Decease, n(\%) & p \\
\hline Endoscopy results & Erosive gastritis & $6(6.3 \%)$ & $2(12.5 \%)$ & 0.324 \\
& Ulcer & $90(94.7 \%)$ & $16(100 \%)$ & 0.999 \\
& Esophagitis & $10(10.5 \%)$ & $0(0 \%)$ & 0.352 \\
& Varices & $8(8.4 \%)$ & $3(18.8 \%)$ & 0.195 \\
Forest clasification & Other & $2(2.1 \%)$ & $0(0 \%)$ & 0.999 \\
& 1A & $1(1.1 \%)$ & $0(0 \%)$ & 0.091 \\
& 1B & $7(7.4 \%)$ & $2(12.5 \%)$ & \\
& 2A & $19(20 \%)$ & $6(37.5 \%)$ & \\
& 2C & $10(10.5 \%)$ & $3(6.3 \%)$ & \\
\end{tabular}

Table 5. Logistic Regression Analysis of the Factors Affecting Mortality

\begin{tabular}{|c|c|c|c|c|c|c|c|c|c|c|}
\hline \multirow[b]{3}{*}{ Age } & \multicolumn{5}{|c|}{ Univariate Model } & \multicolumn{5}{|c|}{ Multivariate Model } \\
\hline & OR & & $95 \% \mathrm{CI}$ & & $\mathrm{p}$ & OR & & $95 \% \mathrm{CI}$ & & $\mathrm{p}$ \\
\hline & 3.18 & 1.77 & - & 5.69 & 0.000 & 2.09 & 1.05 & - & 4.15 & 0.035 \\
\hline Comorbidity & 29.02 & 3.77 & - & 223.63 & 0.001 & 9.33 & 1.06 & - & 82.20 & 0.044 \\
\hline Aspirin Use & 2.93 & 1.14 & - & 7.53 & 0.025 & & & & & \\
\hline BUN $\otimes 70$ & 3.09 & 1.28 & - & 7.48 & 0.012 & & & & & \\
\hline RDW & 1.41 & 1.16 & - & 1.72 & 0.000 & 1.30 & 1.05 & - & 1.61 & 0.017 \\
\hline Lymphocytes & 0.56 & 0.35 & - & 0.89 & 0.014 & & & & & \\
\hline
\end{tabular}




\begin{tabular}{|c|c|c|c|c|c|c|c|c|c|c|}
\hline NLR & 1.18 & 1.07 & - & 1.31 & 0.001 & 1.13 & 1,00 & - & 1.28 & 0.041 \\
\hline RDW/Platelet & 728.77 & 1.97 & - & $>1000$ & 0.029 & & & & & \\
\hline Blatchford Score & 1.17 & 1.05 & - & 1.31 & 0.006 & & & & & \\
\hline
\end{tabular}

IQR: Interquartile range, HGB: Heamoglobin, RDW: red blood cell distribution width, NLR:neutrophil/lymphocyte ratio

Multivariate logistic regression analysis showed that age, comorbidities and RDW and NLR values were independently and significantly correlated with mortality rates $(\mathrm{p}<0.05)$ (Table 5).

ROC analysis was performed to determine the predictive value of NLR (Figure 1) and RDW (Figure 2) for mortality.

NLR values were significantly effective in distinguishing between deceased patients and survivors (area under the curve 0.717 [0.606-0.829]). A cut-off value of 3 was observed to be the highest cut-off value for NLR, (area under the curve) in distinguishing between deceased patients and survivors. Using this cut-off value, sensitivity was $71.4 \%$, specificity was $37.7 \%$, positive predictive value was $60.2 \%$ and negative predictive value was $86.2 \%$. RDW values were significantly effective in distinguishing between deceased patients and survivors (Area under the curve 0.726 [0.609-0.8243]). A cut-off value of 14.5 was observed to be the highest cut-off value of RDW (area under the curve) in distinguishing between deceased patients and survivors. Using this cut-off value, sensitivity was $67.9 \%$, specificity was $74.7 \%$, positive predictive value was $47.5 \%$ and negative predictive value was $87.3 \%$.

\section{DISCUSSION}

Patients presenting with upper GI bleeding show high mortality rates and $80 \%$ of these deaths occur from bleeding-related causes. Other leading causes of mortality include terminal malignancy, multiorgan failure and cardiopulmonary decompensation $(8,9)$.

In our study, age, comorbidities, as well as NLR and RDW values were observed to be independent risk factors affecting 30-day mortality rates. Neutrophils and lymphocytes increase secondary to GI bleedinginduced inflammationGI. Kandemir et al. reported that leukocytes and NLR were effective predictors of mortality in patients with GI bleeding (10). Peng et al. reported the prognostic efficacy of NLR in patients with GI bleeding (11). In our study, the neutrophil count was high, the lymphocyte count was low, and the NLR ratio was high in deceased patients. In our view, the high neutrophil rate and NLR were associated with more severe inflammation in deceased patients.

Isik et al. reported that RDW was increased in patients with GI bleeding and that RDW values were higher in deceased patients than in survivors. This observation was attributed to increased stress in patients (12). Ozdemir et al. reported that among patients followedup in the intensive care unit, RDW was lower in patients who were discharged and higher in deceased patients (13). In our study, RDW was higher, the PLT count was similar and the RDW/ PLT ratio was higher in deceased patients, which can be attributed to the fact that RDW was elevated secondary to increased stress.

A study reported by Christensen et al. showed that the 30-day mortality rate was $10.8 \%$ in patients with bleeding peptic ulcers (14). The 30-day mortality rate in patients with upper GI bleeding was $4.5 \%$ in a study reported by Marmo et al. GI (15), $6.2 \%$ in a study reported by Sung et al. (8), $8.7 \%$ in a study reported by Lee et al. (16), and $31.1 \%$ in a study reported by Crooks et al. (17). In our study, the 30-day mortality rate was $14.4 \%$ (higher than rates reported in the literature), which could be attributed to several factors ranging from social characteristics of patients to the treatment administered. Additionally, it might be related to the fact that patients with mild disease were not referred to our hospital (a tertiary center).

Previous studies have reported that the incidence of upper GI bleeding is higher in elderly patients and it is associated with a higher mortality rate in this age group $(15,18)$. A study reported by Sung et al. investigated mortality patterns in those with upper GIS bleeding over a 30-day period, and reported that deceased patients were older (8). Christensen et al. reported that age was a significant and independent factor associated with 30-day mortality rates in patients with complicated peptic ulcers(ulcers associated with bleeding and/ or perforation) (14). In our study, the mean age of patients was $58.2 \pm 17.3$ years. We observed that the mean age was higher in deceased patients, and that age was inde- pendantly associated with mortality rates, which could be attributed to increased vascular fragility, decreased cardiac reserve, increased comorbidities and drug use in elderly patients.

A few studies have reported that upper GI bleeding is more common in men $(15,18)$. However, Marmo et al. (15) and Sung et al. (8) reported no correlation between the 30-day mortality rates and sex. Our study results were consistent with the literature in that upper GIS bleeding was observed more commonly in men, although no significant correlation was observed between mortality rates and sex. In our view, upper GI bleeding occurs more commonly in men because the use of substances such as tobacco and alcohol, as well as antiplatelet and anticoagulants drugs is greater in men secondary to higher occurrence of ischemic events and a more stressful life. However, a patient's clinical condition affects mortality, and a patient's sex is not relevant in this context.

Previous studies have reported that patients most commonly present with melena, although no correlation was observed between melena and mortality rates (19, 20). Lanas et al. reported that patients with hematemesis were at a higher risk of death (21). In our study, the most common symptom observed in patients was melena; however, melena was not correlated with mortality rates. In our opinion, melena was the most common symptom in our study because even small quantities of bleeding $(50 \mathrm{~mL})$ cause melena. A small quantity or slow chronic bleeding from a lower site in the GI can cause melena. However, we reckon that symptom severity and the quantity of bleeding affect mortality rates rather than the occurrence of bleeding.

A few studies have reported that comorbidities and the administration of certain drugs increase the incidence of GI $(19,22$, 23). Siddique et al. reported that comorbidities such as stroke and pneumonia were not associated with readmission and mortality; however, cirrhosis and malignancy, among other such conditions, increase the incidence of re-bleeding (24). Sung et al. reported high 30-day mortality rates in patients with multiple comor- 
bidities and predisposing factors (use of nonsteroidal anti-inflammatory drugs [NSAIDs] and/or aspirin)(8). Marmo et al. reported a higher occurrence of comorbidities in patients who died within 30 days of developing upper GI bleeding (15). Lanas et al. reported that the mortality risk was lower in patients who used warfarin and NSAIDs (21). In our study, mortality rate was high in patients with comorbidities and in those with malignancy and cirrhosis; however, predisposing factors did not affect mortality rates. The 30 -day mortality rate might be higher in patients with cirrhosis and malignancies because these conditions precipitate bleeding. Cirrhosis causes bleeding secondary to variceal bleeding and reduction in serum levels of coagulation factors is observed in both malignancy and cirrhosis. We concluded that antiplatelet and anticoagulant agents did not affect the 30-day mortality because their doses were adjusted in this study.

Reportedly, hemodynamic instability is a common factor associated with upper GI bleeding-induced mortality $(21,25,26)$. Marmo et al. reported that heart rate was higher and blood pressure was lower at the time of admission to the hospital in patients who died within 30 days of developing upper GI bleeding (15). In our study, we observed significant hypotension and higher pulse rate in deceased patients, although this difference was statistically non-significant. İt is known that blood pressure reduction is proportionate to bleeding and tachycardia is known to occur in patients with severe bleeding. This non-significant result might be attributed to a small number of deceased patients and the use of drugs causing bradycardia.

Onder et al. reported decreased serum HGB levels in deceased patients with upper GI (18). Marmo et al. reported lower serum HGB levels in patients who died within 30 days (15). Increased BUN values observed in patients with GI bleed- ing are caused by increased re-absorption and hypovolemia following conversion of blood proteins to urea by intestinal bacteria (27). Tomizawa et al. observed that patients with serum $\mathrm{BUN}$ values $>21 \mathrm{mg} / \mathrm{dL}$ showed a higher incidence of GI bleeding (28). In our study, serum HGB and BUN levels were significantly higher in deceased patients. These results can be explained by the fact that bleeding is an important factor that alters patients' hemodynamics and is associated with mortality. It is reasonable to deduce that hypovolemic shock led to higher BUN values in patients with more severe bleeding.

Sung et al. reported a correlation between the 30-day mortality rates and lesion localization (8). Crooks et al. reported similar mortality rates regardless of lesion localization (17). In our study, we could not identify a correlation between mortality rates and lesion localization. In our opinion, although the quantity of bleeding is affected by several factors including vascularization in the lower parts of lesions, lesions in specific locations (esophageal varices), and lesion invasion, these factors did not affect mortality rates because bleeding ceased after the administration of treatment. Additionally, investigating a small number of patients with lesions in only a few locations might lead to inaccurate and non-significant results.

Marmo et al. reported that ulcer diameter was wider in patients who died within 30 days (15). Sung et al. reported that the mortality rate was higher in patients with active bleeding ulcers (8). Sey et al. reported mortality rates of $7 \%$ in patients with esophageal and gastric lesions and $10 \%$ in those with duodenal bleeding (4). In our study, most patients were classified into grade 3 based on the Forrest classification and no correlation was observed between mortality rates and the Forrest classification. However, deceased patients showed a higher Glasgow-Blatchford score. We consider quantity of bleeding to be a primary determinant of mortality rather than the features of the bleeding lesion. Therefore, theGlasgow-Blatchford score (which predicts the need for hemodynamic intervention) is more useful than the Forrest score. The lack of a significant correlation between mortality rates and the Forrest score could be attributed to the fact that active bleeding causes severe and acute blood loss, whereas slow chronic bleeding causes severe losses over time. Additionally, a small number of patients with stage 1 and stage 2 disease who were investigated might have led to this result.

\section{CONCLUSION}

In conclusion, several factors affect 30-day mortality rates in patients with GI bleeding. Clinicians should be aware that elderly patients, those with comorbidities and impaired hemodynamics require careful and long-term follow-up and that these patients are at a high risk for mortality.

\section{LIMITATIONS}

Following are the limitations of this study: (1) This was a small-scale study. (2) Mortality was attributed only to GI bleeding. (3) Comorbidities and drugs used were evaluated based only on the patients' medical history without analysing documented data regarding the duration of disease and drug use. (4) Although eradication of Helicobacter pylori decreases bleeding in patients with peptic ulcer, we could not analyse the presence of this organism in our study population.

\section{CONFLICT OF INTEREST}

The authors declare no conflict of interest.

\section{REFERENCES}

1. Vreeburg E, Terwee C, Snel P, Rauws E, Bartelsman J, VdMeulen J, et al. Validation of the Rockall risk scoring system in upper gastrointestinal bleeding. Gut 1999;44(3):331-5.

2. Yalçin MS, Kara B, Öztürk NA, Ölmez S, Tasdogan BE, Tas A. Epidemiology and Endoscopic Findings of the Patients Suffering from Upper Gastrointestinal System Bleeding. Dicle Tip Derg 2016;43(1):73.

3. Szura M, Pasternak A. Upper non-variceal gastrointestinal bleeding-review the effectiveness of endoscopic hemostasis methods. World J Gastrointest Endosc 2015;7(13):1088.

4. Sey MSL, Mohammed SB, Brahmania M, Singh S, Kahan BC, Jairath V. Comparative outcomes in patients with ulcer vs nonulcer related acute upper gastrointestinal bleeding in the United Kingdom: a nationwide cohort of 4478 patients. Aliment Pharmacol Ther. 2019: 49(5):537-545

5. Abougergi MS, Travis AC, Saltzman JR. The in-hospital mortality rate for upper GI hemorrhage has decreased over 2 decades in the 
United States: a nationwide analysis. Gastrointest Endosc 2015;81(4):882-8. e1.

6. Wuerth BA, Rockey DC. Changing Epidemiology of Upper Gastrointestinal Hemorrhage in the Last Decade: A Nationwide Analysis. Dig Dis Sci 2018;63(5):1286-93.

7. Cander B, Ertekin B, Hasan K, Mehmet G, Dündar D, Koçak S, et al. Factors Affecting The Length of Hospitalization at The Patients Admit to Emergency Department With Gastrointestinal Bleeding. Firat Tip Derg 2011;16(2):051-4.

8. Sung JJ, Tsoi KK, Ma TK, Yung MY, Lau JY, Chiu PW. Causes of mortality in patients with peptic ulcer bleeding: a prospective cohort study of 10,428 cases. Am J Gastroenterol 2010;105(1):84-9.

9. Saeed ZA, Ramirez FC, Hepps KS, Cole RA, Graham DY. Prospective validation of the Baylor bleeding score for predicting the likelihood of rebleeding after endoscopic hemostasis of peptic ulcers. Gastrointest Endosc 1995;41(6):561-5.

10. Kandemir A, Süle M, Yavaşoğlu İ, Türe M, Coşkun A, Yükselen A, et al. Assessment of the Relationship Between Leukocyte Count and Neutrophil-to-Lymphocyte Ratio and Clinical Course in Non-Variceal Upper Gastrointestinal System Bleeding. SakaryaTip Dergisi 2018;8(2):320-6.

11. Peng Z, He Z, Guo X, Tacke F, Yang S-S, Sugawara Y, et al. Neutrophil to lymphocyte ratio and albumin-bilirubin score for predicting the in-hospital mortality of hepatocellular carcinoma with acute upper gastrointestinal bleeding. AME Med J 2017;2(11).

12. Isik B, Yilmaz MS, Yel C, Kavalci C, Solakoglu GA, Özdemir M, et al. Importance of red blood cell distribution width (RDW) in patients with upper gastrointestinal haemorrhage. JPMA 2016;66(2):151-4.

13. Özdemir R, Mutlu NM, Özdemir M, Akcay M, Yel C, TURAN IÖ. The importance of red cell distribution width (RDW) in patient follow up in intensive care unit (ICU). Acta Med Mediter 2016;32:349-54.

14. Christensen S, Riis A, Norgaard M, Sorensen HT, Thomsen RW. Short-term mortality after perforated or bleeding peptic ulcer among elderly patients: a population-based cohort study. BMC Geriatr 2007;7:8.

15. Marmo R, Koch M, Cipolletta L, Bianco MA, Grossi E, Rotondano G, et al. Predicting mortality in patients with in-hospital nonvariceal upper GI bleeding: a prospective, multicenter database study. Gastrointest Endosc 2014;79(5):741-9 e1.

16. Lee YJ, Min BR, Kim ES, Park KS, Cho KB, Jang BK, et al. Predictive factors of mortality within 30 days in patients with nonvariceal upper gastrointestinal bleeding. Korean J Intern Med 2016;31(1):54-64.

17. Crooks C, Card T, West J. Reductions in 28-day mortality following hospital admission for upper gastrointestinal hemorrhage. Gastroenterology 2011;141(1):62-70.

18. Önder A, Kapan M, Taşkesen F, Aliosmanoğlu İ, Arıkanoğlu Z, Gül M, et al. Risk factors in non-variceal and non-malignancy upper gastrointestinal system bleedings in the patients undergoing surgery. Turk J Surg 2011;27(4):216-21.

19. Gölgeli H, Ecirli Ş, Kutlu O, Başer H, Karasoy D. Evaluation of the patients that followed up for upper gastrointestinal system bleeding. Dicle Med J 2014; 41: 495-501.

20. Adamopoulos AB, Baibas NM, Efstathiou SP, Tsioulos DI, Mitromaras AG, Tsami AA et al. Differentiation between patients with acute upper gastrointestinal bleeding who need early urgent upper gastrointestinal endoscopy and those who do not. A prospective study. Eur J Gastroenterol Hepatol 2003;15: 381-7.

21. Lanas A, Aabakken L, Fonseca J, Mungan ZA, Papatheodoridis GV, Piessevaux H, et al. Clinical predictors of poor outcomes among patients with nonvariceal upper gastrointestinal bleeding in Europe. Aliment Pharmacol Ther 2011;33(11):1225-33.

22. Thomopoulos KC, Vagenas KA, Vagianos CE, Margaritis VG, Blikas AP, Katsakoulis EC, et al. Changes in aetiology and clinical outcome of acute upper gastrointestinal bleeding during the last 15 years. European J Gastroenterol Hepatol 2004;16(2):177-82.

23. Baradaran F, Norouzi A, Tavassoli S, Baradaran A, Roshandel G. Factors associated with outcome in patients with acute upper gastrointestinal bleeding in a tertiary referral center in Northern Iran. Middle East J Dig Dis 2016;8(3):201.

24. Siddique SM, Mehta SJ, Lewis JD, Neuman MD, Werner RM. Rates of hospital readmission among Medicare beneficiaries with gastrointestinal bleeding vary based on etiology and comorbidities. Clin Gastroenterol Hepatol 2019;17(1):90-7. e3.

25. Lahiff C, Shields W, Cretu I, Mahmud N, McKiernan S, Norris S, et al. Upper gastrointestinal bleeding: predictors of risk in a mixed patient group including variceal and nonvariceal haemorrhage. Eur J Gastroenterol Hepatol 2012;24(2):149-54.

26. Jung SH, Oh JH, Lee HY, Jeong JW, Go SE, You CR, et al. Is the AIMS65 score useful in predicting outcomes in peptic ulcer bleeding? World J Gastroenterol 2014;20(7):1846-51.

27. Chalasani N, Clark WS, Wilcox M. Blood urea nitrogen to creatinine concentration in gastrointestinal bleeding: a reappraisal. Am J Gastroenterol 1997;92(10):1796-9

28. Tomizawa M, Shinozaki F, Hasegawa R, Shirai Y, Motoyoshi Y, Sugiyama T, et al. Patient characteristics with high or low blood urea nitrogen in upper gastrointestinal bleeding. World J Gastroenterol 2015;21(24):7500-7505 